\title{
Universal Electronic Cash
}

\author{
Tatsuaki Okamoto Kazuo Olita \\ NTT Laboratorics \\ Nippon Telcgraph and Telephone Corporation \\ 1-2356, Talie, Yoliosulia-shi, Kanagawa-ken, 238-03 Japan
}

\begin{abstract}
This paper proposes the first idcal untraccable electronic cash system which solves the most crucial problem inherent with real cash and all previous untraceable clcctronic cash systems. The main advantage of the new system is that the customer can subdivide his cash balance, $C$ (dollars), into many pieces in any way he pleases until the total value of all subdivided piece cquals $C$. This system can be implemented efficiently. In a typical implementation, the data size of one piece of clectronic cash is less than 100 bytes regardless of the face value of piece, the computation time for each transaction is several seconds, assuming the existence of a Rabin scheme chip. The sccurity of this scheme relies on the difficulty of factoring.
\end{abstract}

\section{Introduction}

Elcctronic cash is one of the most important applications of modern cryptology because an electronic moncy (cash) system will be widely installed in the near future; smart cards will become electronic wallets storing electronic cash. The security of real cash heavily depends on physical propertics such as the dificulty of reproducing bills and coins. The security of electronic cash systems cannot depend on any physical condition, but must be guarantecd by mathematics. Here, cryptographic techniques are essentially uscd to guarantee sccurity. Then, information itself has a value, and electronic cash can be transfered through networks.

What then is the ideal cash system? The criteria describing the ideal cash system are as follows:

(a) Independence: The security of electronic cash cannot depend on any physical condition. Then the cash can be transfered through networks.

(b) Sccurity: The ability to copy (reuse) and forge the cash must be prevented.

(c) Privacy (Untraceability): The privacy of the user should be protected. That is, the relationship between the user and his purchases must be untraccable by anyone.

(d) Off-line payment: When a uscr pay the elcctronic cash to a shop, the procedure between the user and the shop should be exccuted in an off-line manner. That is, the shop does not need to be linked to the host in uscr's payment procedure. 
(e) Transferability: The cash can be transfered to other users.

(f) Dividability: One issued piece of cash worth value $C$ (dollars) can be subdivided into many pieces such that each subdivided piece is worth any desired value less than $C$ and the total value of all pieces is equivalent to $C$.

Several electronic cash systems have been proposed by [Ch, Da, PW, EGY, OkOh2, CFN, OkOh1]. The security of the clectronic cash system by [EGI'] depends on a physical condition. Therefore, [EGY] does not satisfy critcrion (a). There are two types of electronic cash systems satisfying criteria (a), (b) and (c); on-line untraccable clectronic cash systems, and off-line untraceable electronic cash systems.

Some on-line untraceable clectronic cash systems have been proposed by [Ch, Da, PW], which satisfy critcria (a) through (f) except criterion (d). However, the on-line cash systems are not practical from the vicwpoints of turn-around-time, communication cost, and databasc-maintainance cost. Therefore, the off-line cash systems are prefcrable from the practical viewpoint, although they are technically difficult to construct.

An off-line untraceable electronic cash system satisfying criteria (a), (b), (c) and (d) was firstly proposed by [CFN], based on the cut-and-choose methodology and a collision free one-way function technique. An electronic cash system satisfying criteria (a), (b), (c), (d) and (c) was then proposed by [OkOh1]. In [OkOh1], the disposable zcro-knowledge authentication scheme is used in place of the collision free function technique in [CFN].

In $[\mathrm{OkOh} 1]$, an electronic coupon ticket system was also proposed, in which one picce of electronic cash can be subdivided into many pieces whose values are all cquivalent. In this system, however, if a customer pays for an article witl 1 cents, the store receives an enormous number of one-cent elcctronic coupon tickets from the customer (for example, when the price of the article is $\$ 356.27$, the store reccives 35627 electronic coupon tickets, where the data size of each ticket is several kilobytes. So, the storc reccives about 200 megabytes of data for purchasing just one article.) Therefore, no electronic cash system satisfying criterion (f) as well as the other criteria (a) through (c) has been proposed so far.

It must be noted that even the real cash system cannot satisfy criterion (f). This is the reason why we must hold many bills and coins in our wallets. On the other hand, other typical exchange systems such as bank notes and credit cards do not satisfy critcria (a) and (c). Prepaid cards such as telephone cards do not satisfy criterion (a), although they almost satisfy critria (b) through (f). Therefore, we do not have the ideal cash system so far, either electronic or real.

In this paper, we propose the first electronic cash system that satisfies all six criteria. That is, this system is the first version of the idcal cash system. Morcover, the ncw system is more efficient and practical than any previous system even if we restrict the comparison wo the two criteria (a) through (d).

Our scheme uses the cut-and-choose methodology as all previous schemes. The new key techniques of our scheme are the square root molulo $N$ ( $N$ is the Williams integer), and the hierarchical structure table. The former is used mainly for critcria (a) through (e) (or in place of the techniques such as the collision free function [CFN], and disposable zero-knowledge authentication [OkOh1]. The latter combined with the former is used for criterion (f), where the hierarchical structure table corresponds to the structure of the cash system.

This paper is constructed as follows: First, in section 2, we will introduce the background of the key techniques including the number theoretic conventions, and the hicrar- 
chical structure table of the cash systcm. In section 3, we will propose the basic version of our electronic cash systcm. Section 4 explains how electronic credits can be transfered to another customer. Section 5 estimates the properties of the clectronic cash system.

\section{Preparations}

\subsection{Number Theoretic Conventions}

Definition 2.1 $N$ is called the Blum integer [BI] if $N=P Q(P, Q$ are primc) and $P=3$ $(\bmod 4)$, and $Q=3(\bmod 4)$.

$N$ is called the TVilliams integer [W] if $N=P Q(P, Q$ are primc) and $P=3(\bmod 8)$, and $Q=7(\bmod 8)$. Note that the Williams interger is a specific type of the Blum intcger. So, the Williams integer has all properties of the Blum integcr.

Let $(x / N)$ denote the Jacobi symbol, when $N$ is a composite number, and denote the Legendre symbol, when $N$ is a prime. When $N=P Q(P, Q$ are prime), we can classify $Z_{N}^{*}$ into four classes; $Z_{(1,1)}=\left\{x \in Z_{N}^{*} \mid(x / P)=1,(x / Q)=1\right\} Z_{(1,-1)}=\{x \in$ $\left.Z_{N}^{*} \mid(x / P)=1,(x / Q)=-1\right\}, Z_{(-1,1)}=\left\{x \in Z_{N}^{*} \quad \mid(x / P)=-1,(x / Q)=1\right\}$, and $Z_{(-1,-1)}=\left\{x \in Z_{N}^{*} \mid(x / P)=-1,(x / Q)=-1\right\}$.

Clearly, $Z_{(1,1)}$ denotes the set of quadratic residuc intergers in $Z_{N}^{*}$. Hercafter, we often write $Q R_{N}$ as $Z_{(1,1)}$, and $Q N R_{N}$ as the other classes.

Proposition 2.2 Let $N$ be the Blum integer, and $x \in Q R_{N}$. Then, for any integer $t$ $(1 \leq t)$, there are four values $y_{1}, y_{2}, y_{3}, y_{4}$ such that $y_{i}^{2^{t}} \equiv x(\bmod N)$ and that $y_{1} \in Z_{(1,1)}$, $y_{2} \in Z_{(1,-1)}, y_{3} \in Z_{(-1,1)}, y_{1} \in Z_{(-1,-1)}$.

In addition, $y_{1} \equiv-y_{4}(\bmod N), y_{2} \equiv-y_{3}(\bmod N),\left(y_{1} / N\right)=\left(y_{4} / N\right)=1$, and $\left(y_{2} / N\right)=\left(y_{3} / N\right)=-1$.

The above proposition immediately implies that four values of $2^{t}$-th root $y$ of $x$ can be uniquely determined by two bit information; one is whether $(y / N)=1$ or -1 , and the other is whether $y<N / 2$ or not. In other words, when $y<N / 2$, there are two valucs of $y$, one of which is $(y / N)=1$ and the other is $(y / N)=-1$.

$x^{1 / 2^{t}} \bmod N(1 \leq t)$ can be computed efficiently (in expected polynomial timc) from $x, P, Q[\mathrm{R}, \mathrm{Ber}]$, and $(y / N)$ can also be computed efficiently from $y$ and $N$, while to compute $x^{1 / 2^{2}} \bmod N$ from $x$ and $N$ is as difficult as factoring $N$ [R].

Proposition 2.3 Let $N=P Q$ be the Williams integer. Then, for any $x \in Z_{N}^{*}$, either one of $x,-x, 2 x$ and $-2 x$ is in $Q R_{N}$. In addition, when $a x \in Q R_{N}$ ( $a$ is either $1,-1,2$, or -2$), b x$ is not in $Q R_{N}(b \neq a$, and $b$ is either $1,-1,2$, or -2$)$.

The above proposition is easily proven by the following result;

$$
(-1 / P)=-1,(-1 / Q)=-1,(2 / P)=-1,(2 / Q)=1 .
$$

Definition 2.4 Let $N$ be the Williams integer, and $x \in Q R_{N}$.

$$
\left[x^{1 / 2^{r}} \bmod N\right]_{Q R}=y
$$


such that $y^{2^{t}}=x \bmod N$ and $y \in Q R_{N} .(1 \leq t)$

$$
\left[x^{1 / 2^{t}} \bmod N\right]_{1}=y^{\prime}
$$

such that $y^{\prime 2^{t}}=x \bmod N,\left(y^{\prime} / N\right)=1$ and $0<y^{\prime}<N / 2 .(1 \leq t)$

$$
\left[x^{1 / 2^{\mathrm{t}}} \bmod N\right]_{-\mathrm{l}}=y^{\prime \prime}
$$

such that $y^{\prime 2^{\prime}}=x \bmod N,\left(y^{\prime \prime} / N\right)=-1$ and $0<y^{\prime \prime}<N / 2 .(1 \leq t)$

Let $N$ be the Williams integer, and $z \in Z_{N}^{*}$.

$$
<z>_{Q R}=d z \bmod N
$$

such that $d \in\{ \pm 1, \pm 2\}$ and $d z \bmod N \in Q R_{N}$.

$$
<z>_{1}=d^{\prime} \approx \bmod N
$$

such that $d^{\prime} \in\{1,2\}$ and $\left(d^{\prime}=/ N\right)=1$.

$$
<z>_{-1}=d^{\prime \prime} z \bmod N
$$

such that $d^{\prime \prime} \in\{1,2\}$ and $\left(d^{\prime \prime} z / N\right)=-1$.

From the properties of the Williams number (and the Blum number), each value of $y, y^{\prime}, y^{\prime \prime}, d, d^{\prime}, d^{\prime \prime}$ is uniquely determined respectively.

\subsection{Hierarchical Structure Table}

In our cash system, the hierarchical structure table plays an important role becausc it allows the issued electronic bill $C$ to be subdivided into many pieces such that each subdivided piece is worth any desired value less than $C$ and the total value of all pieces is equivalent to $C$.

The hierarchical structure table is a trce of $t$ levels, in which each node has two sons, the unique root node exists at the top of the tree. So, there are $2^{i-1}$ nodes at the $i$-th level.

Here, we show the significance of the tree in our cash system. For easy understanding, we use a simple example, where the tree has three levels, and the value of the issued bill $C$ is $\$ 100$. The nodes of the $i$-th level correspond to $\$ 100 / 2^{i-1}$. So, the customer can use the bill in $\$ 25$ increments, since the nodes of the bottom level (the third level) correspond to $\$ 25$ (see Figure 1).

We give two restrictions to the usage of the bill with rclating to the tree as follows:

1. The value corresponding to a node, $N$, is the total of the values corresponding to nodes that are the direct sons of $N$.

2. When a node (the corresponding value) is used, all descenciant nodes and all ancestor nodes of this node cannot be used.

3. No node can be used morc than once. 
We show the case when customer Alice uses $\$ 75$ first and then uses $\$ 25$. When she uses $\$ 75$, she must use node $\Gamma_{00}(\$ 50)$, and node $\Gamma_{010}(\$ 25)$. From the above restrictions, only $\Gamma_{011}(\$ 25)$ can be used aiter the use of $\Gamma_{00}$ and $\Gamma_{010}$ (see Figure 2).

More gencrally, if Alice wants to use a bill worth $\$ 1000$ by the cent, she would need a hierarchical structure table of 17 levels $\left(\log _{2} 100000 \approx 16.5\right)$. She would then use about 8 nodes in average (minimum: one node; maximum: 16 nodes) in order to pay by the cent for each purchase (c.g., \$334.36 payment).

Morcover, in our concrete cash scheme that will be shown in the following scctions, we need two hierarchical structure tables ( $\Gamma$ table and $\Lambda$ tablc); $\Gamma$ table is used to rcalize the first restriction, and $\Lambda$ table to realize the second restriction. $\Gamma$ table and $\Lambda$ table have the same structure such that they are trees with the same topology (or the same number of layers), and that $\Gamma_{j_{1} \ldots j_{t}}$ and $\Lambda_{j_{1} \ldots j_{t}}$ both correspond to the same position node $\left(\right.$ Node $\left._{j_{1} \ldots j_{\ell}}\right)$ of the money structure table. In the example of Figures 1 and $2, \Gamma_{00}$ and $\Lambda_{00}$ correspond to the same position node, the lelt node of $\$ 50$, of the money structure table.

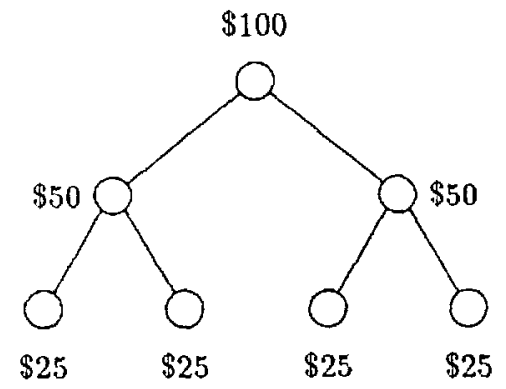

Figure 1: Hicrarchical Structure Table (Moncy Structure)

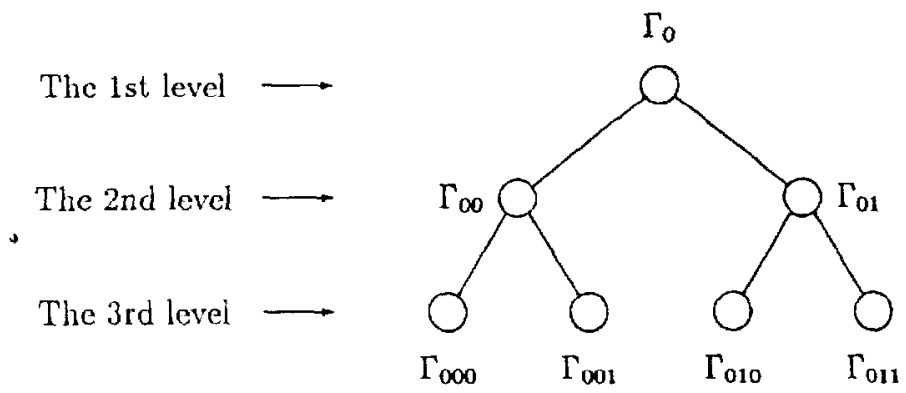

Figure 2: Hierarchical Structure Table ( $\Gamma$ Table) 


\section{Basic Universal Electronic Cash Scheme}

In this section, we introduce the basic universal electronic cash sclieme which satisfics the five criteria ((a) through (f) except (c)(Transfcrability)).

\subsection{Protocol}

\section{Protocol 1 (Basic universal electronic cash):}

For blind digital signatures[Ch], bank $A$ has generated keys of the RSA scheme; $\left(e_{A}, n_{A} ; d_{A}\right),\left(e_{A}^{\prime}, n_{A}^{\prime} ; d_{A}^{\prime}\right),\left(e_{A}^{\prime \prime}, n_{A}^{\prime \prime} ; d_{A}^{\prime \prime}\right), \ldots$, where $\left(e_{A}, n_{A}\right),\left(e_{A}^{\prime}, n_{A}^{\prime}\right), \ldots$ are public keys, and $d_{A}, d_{A}^{\prime}, \ldots$ are the corresponding secret keys. $A$ has published $\left(e_{A}, n_{A}\right),\left(e_{A}^{\prime}, n_{A}^{\prime}\right),\left(e_{A}^{\prime \prime}\right.$, $\left.n_{A}^{\prime \prime}\right), \ldots$, where $\left(e_{A}, n_{A}\right)$ corresponds to the electronic license that $A$ issucs, and $\left(\epsilon_{A}^{\prime}, n_{A}^{\prime}\right)$, $\left(e_{A}^{\prime \prime}, n_{A}^{\prime \prime}\right), \ldots$ correspond to the value of the clcctronic bill that $A$ issues. For example, $\$ 100$ corresponds to $\left(e_{A}^{\prime}, n_{A}^{\prime}\right)$, and $\$ 500$ corresponds to $\left(e_{A}^{\prime \prime}, n_{A}^{\prime \prime}\right)$, ctc. Bank $A$ also scts the security parameter $K=O\left(\left|n_{A}\right|\right)=O\left(\left|n_{A}^{\prime}\right|\right)=\ldots$ (for example, $\left.K=40\right)$.

$A$ has also published thrce randomized hash functions, $f_{\Gamma}, f_{\Lambda}, f_{\Omega}$, to gencrate the hicrarchical structure tables, $\Gamma$ table and $\Lambda$ table. IIcre, the function values are assumed to distribute uniformly (for example, the universal hash functions [CW], and pscudorandom gencrator). Note that the one-wayness or collision-freeness is not required for these functions.

Customer $P$ has a bank account number $I D_{P}$ and has generated the key of the RSA scheme, $\left(e_{P}, n_{P} ; d_{P}\right)$, and published $\left(e_{P}, n_{P}\right)$ for digital signatures.

Note 1: Any multiple blind digital signaturc [OkOl1]] can be used in place of the RSA scheme for bank $A$ above. For example, the blind digital signature scheme bascd on the Fiat-Shamir signature scheme [OKOh2] can be used for this purpose. Mlorcover, any digital signature scheme can be used in place of the RSA scheme for customer $P$ above. For example, [FOM] can be used for this purpose.

Note 2: The sccure exchange problem is out of the scope of this paper. For example, $A$ and $P$ exchange electronic cash and withdrawal, $P$ and $V$ exchange payment and articles, and $V$ and $A$ exchange payment history and credit. The secure exchange problem can be practically solved by the usage of digital signature sehemes. More secure but less efficient solutions for this problem has been shown in [EGL].

Part I.

When a customer $P$ opens an account at bank $A, A$ issues an clectronic license $B=\left\{B_{i} \mid\right.$ $1 \leq i \leq K / 2\}$ to usc the elcctronic cash of bank $A$. (Preciscly, the electronic license is $\left(B,\left\{I_{i}, N_{i}\right\}, L\right)$. For simplicity, however, we simply call it $B$.) To get $B, P$ conducts the following protocol with $A$. This procedure is exccuted only once when $P$ opens the account, unless $P$ uses the electronic cash invalidly.

Step 1: Customer $P$ chooses a random value $a_{i}$, and the Williams integers $N_{i}$ witl two large prime factors $P_{i}, Q_{i}\left(N_{i}=P_{i} Q_{i}\right)$, where $P_{i} \equiv 3(\bmod 8)$ and $Q_{i} \equiv 7$ $(\bmod 8)$, for $i=1, \ldots, K$.

Step 2: $P$ forms and sends $K$ blind candidates $W_{i}(i=1, \ldots, K)$ to bank $A$.

$$
W_{i}=r_{i}^{* A} g\left(I_{i} \| N_{i}\right) \bmod n_{A} \quad \text { for } 1 \leq i \leq K,
$$


where $r_{i} \in Z_{n_{1}}$ is a random integer, $g$ is an appropriate one-way hash function, and

$$
\begin{gathered}
S_{i}=I D_{P}\left\|a_{i}\right\|\left(g\left(I D_{P} \| a_{i}\right)\right)^{d_{P}} \bmod n_{P}, \\
=S_{1, i} \| S_{2, i}, \\
I_{1, i}=S_{1, i}^{2} \bmod N_{i}, I_{2, i}=S_{2, i}^{2} \bmod N_{i}, \\
I_{i}=I_{1, i} \| I_{2, i} .
\end{gathered}
$$

Here, || denotes the concatenation.

Step 3: $A$ chooses a random subsct of $K / 2$ blind canclidates indices $U=\left\{i_{j}\right\}, 1 \leq i_{j} \leq K$ for $1 \leq j \leq K / 2$ and transmits it to $P$.

Step 4: $P$ displays the $a_{i}, P_{i}, Q_{i},\left(g\left(I D_{P} \| a_{i}\right)\right)^{d p} \operatorname{mor} n_{P}, I D_{P}, r_{i}$ for all $i$ in $U$, then $A$ checks them. If they arc not valid, $A$ halts this protocol. To simplify notations, we will assume that $U=\{K / 2+1, K / 2+2, \ldots, K\}$.

Step 5: $A$ gives $P$

$$
\left(\prod_{i=1}^{K / 2} W_{i}\right)^{d_{A}} \bmod n_{A}
$$

Step 6: $P$ can then extract the clectronic license $B=\left(\prod_{i=1}^{K / 2} g\left(I_{i} \| N_{i}\right)\right)^{d_{A}} \bmod n_{A}$.

\section{Part II.}

When customer $P$ wants bank $A$ to issue an eicctronic bill worth $\$ 100, C$, which corresponds to $\left(e_{A}^{\prime}, n_{A}^{\prime}\right), P$ conducts the following protocol with $A$.

Step 1: $P$ chooses a random valuc $b$, forms and sends $Z$ to $A$.

$$
Z=r^{e^{\prime}} g(B \| b) \bmod n_{A}^{\prime}
$$

where $r \in Z_{n_{A}^{\prime}}$ is a random integer.

Step 2: $A$ gives $Z^{d^{\prime}}{ }_{A} \bmod n_{A}^{\prime}$ to $P$ and charges $P$ 's account $\$ 100$.

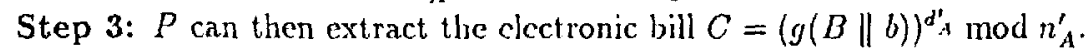

\section{Part III.}

To pay a shop $V$ a certain amount of moncy, $P$ and $V$ proceed as follows:

First, for easy understanding, we will show a simple example of this protocol, when $P$ pays $\$ 75$ to $V$ based on the hierarchical structure table of thrce levels, as was shown in subsection 2.2. Here, we assume that $P$ has received $\$ 100$ bill $C$ from Bank $A$ in Part II.

Step 1: As the preliminary stage of Part III, $P$ computes the value of $\Gamma_{i, 0}(i=1, \ldots, K / 2)$ as follows:

$$
\Gamma_{i, 0}=<f_{\Gamma}\left(C\|0\| N_{i}\right)>_{Q R} .
$$

(See Subsection 2.1 for the notation of $<>_{Q R^{*}}$ )

Step 2: When $P$ decides to pay $\$ 75$, first $P$ computes $X_{i, 00}$ (corresponding to $\$ 50$ ) and $X_{i, 010}$ (corresponding to $\left.\$ 25\right)(i=1, \ldots, K / 2)$ as follows:

$$
X_{i, 00}=\left[\Gamma_{i, 0}^{1 / 4} \bmod N_{i}\right]_{-1}
$$




$$
X_{i, 010}=\left[\left(\Omega_{i, 0}^{2} \Gamma_{i, 0}\right)^{1 / 8} \bmod N_{i}\right]_{-1} .
$$

Here, $\Omega_{i, 0}=<f_{\Omega}\left(C\|0\| N_{i}\right)>_{1}$.

$P$ sends $\left(I_{i}, N_{i}, X_{i, 00}, X_{i, 010}\right)(i=1, \ldots, K / 2)$ and $(B, C)$ to $V$.

Note: The above calculation of $X_{i, 00}$ and $X_{i, 010}$ is based on the following algorithm:

$$
\begin{aligned}
X_{i, 00} & =\left[\Gamma_{i, 00}^{1 / 2} \bmod N_{i}\right]_{-1}, \\
X_{i, 010} & =\left[\Gamma_{i, 010}^{1 / 2} \bmod N_{i}\right]_{-1},
\end{aligned}
$$

where

$$
\begin{gathered}
\Gamma_{i, 00}=\left[\Gamma_{i, 0}^{1 / 2} \bmod N_{i}\right]_{Q R} \\
\Gamma_{i, 01}=\left[\Omega_{i, 0} \Gamma_{i, 0}^{1 / 2} \bmod N_{i}\right]_{Q R} \\
\Gamma_{i, 010}=\left[\Gamma_{i, 01}^{1 / 2} \bmod N_{i}\right]_{Q R}
\end{gathered}
$$

Here, summarizing the algorithm, first, the $\Gamma$ table of the correponding nodes $\left(\Gamma_{i, 00}, \Gamma_{i, 010}\right)$ are calculated, then the sqquarc roots of these values in $Q N R$ (these Jacobi symbol values arc -1$)$ are $X_{i, 00}$ and $X_{i, 010}$.

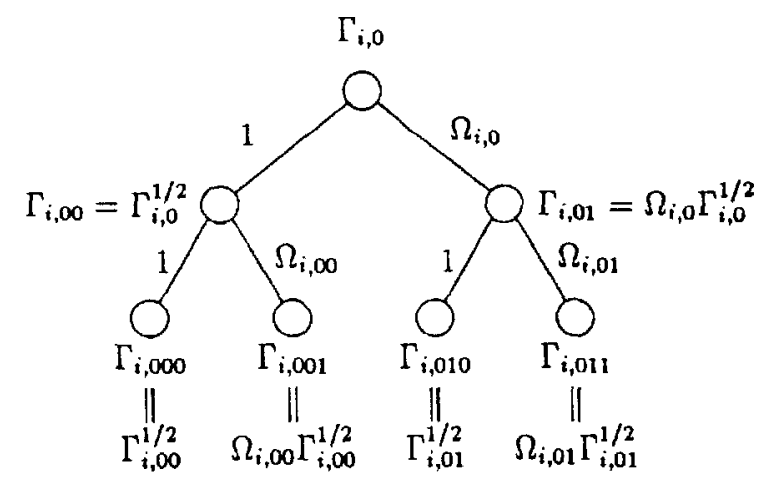

Figure 3: Node Values of $\Gamma$ Table (Three Laycr Example)

Step 3: $V$ verifies the validity of the signatures $B$ for $\left\{\left(I_{i}, N_{i}\right)\right\}$, and $C$ for $B$. $V$ computes $\Omega_{i, 0}, f_{\Gamma}\left(C\|0\| N_{i}\right)$ i.hen verifies the validity of $X_{i, 00}$ and $X_{i, 010}\left(i=1, \ldots, K^{\prime} / 2\right)$ such that

$$
\begin{gathered}
\left(X_{i, 00} / N_{i}\right)=\left(X_{i, 010} / N_{i}\right)=-1, \\
X_{i, 00}^{1}=d_{i} \delta_{\Gamma}\left(C\|0\| N_{i}\right) \bmod N_{i} \\
X_{i, 010}^{8}=d_{i} \Omega_{i, 0}^{2} f_{\Gamma}\left(C\|0\| N_{i}\right) \bmod N_{i},
\end{gathered}
$$

where $d_{i} \in\{ \pm 1, \pm 2\}(i=1, \ldots, K / 2)$. If they are valid, $V$ selects random bits, $E_{i, 00}, E_{i, 010} \in\{0,1\}(i=1, \ldots, K / 2)$, and sends them to $P$. Otherwise $V$ halts this protocol. 
Step 4: $P$ computes

$$
\begin{aligned}
Y_{i, 00} & =\left[\Lambda_{i, 00}^{1 / 2} \bmod N_{i}\right]_{(-1)^{E_{i, 00}}}, \\
Y_{i, 010} & =\left[\Lambda_{i, 010}^{1 / 2} \bmod N_{i}\right]_{(-1)^{E_{i, 010}}},
\end{aligned}
$$

and sends $\left(Y_{i, \infty}, Y_{i, 010}\right)(i=1, \ldots, K / 2)$ to $V$. Here,

$$
\begin{gathered}
\Lambda_{i, 00}=<f_{\Lambda}\left(C\|00\| N_{i}\right)>_{Q R}, \\
\Lambda_{i, 010}=<f_{\Lambda}\left(C\|010\| N_{i}\right)>_{Q R} .
\end{gathered}
$$

Step 5: $V$ vcrifics that

$$
\begin{aligned}
\left(Y_{i, 00} / N_{i}\right) & =(-1)^{E_{i, 00}},\left(Y_{i, 010} / N_{i}\right)=(-1)^{E_{i, 010}}, \\
Y_{i, 00}^{2} & =d_{i}^{\prime} \int_{\Lambda}\left(C\|00\| N_{i}\right) \bmod N_{i}, \\
Y_{i, 010}^{2} & =d_{i}^{\prime \prime} f_{\Lambda}\left(C\|010\| N_{i}\right) \bmod N_{i},
\end{aligned}
$$

where $d_{i}^{\prime \prime}, d_{i}^{\prime \prime} \in\{ \pm 1, \pm 2\}(i=1, \ldots, K / 2)$. If verification succeds, $V$ accepts $P$ 's messages as $\$ 75$ from electronic bill $C$.

Next, we show the protocol of Part III in general cases. IIcre, we assume that $\Gamma$ table has more than $t$ lcvels, and that the node corresponding to the value of $P$ 's payment to $V$ is $\Gamma_{j_{1} \ldots j_{t}}$ (and $\Lambda_{j_{1} \ldots j_{t}}$ ), wherc $j_{1}, \ldots, j_{t} \in\{0,1\}$. Usually, there are several nodes which correspond to the payment (e.g., in the above simple example, two nodes form P's $\$ 75$ payment). Then, the following protocol of cach nocle must be cxecuted simultancously, in the same manner as the above protocol, which has two nodes.

Step 1: This preliminary stage of Part III is the same as the above protocol.

Step 2: When $P$ determines the node, $\Gamma_{j_{1} \ldots j_{\ell}}$ (and $\Lambda_{j_{1} \ldots j_{l}}$ ), corresponding to the payment, $P$ computes $X_{i, j_{1} \ldots j_{i}}$,

$$
X_{i, j_{1} \cdots j_{t}}=\left[\left(\Omega_{i, j_{1} \cdots j_{t-1}}^{2^{t-1} j_{t}} \Omega_{i, j_{1} \cdots j_{t-2}}^{2_{t-2} j_{t-1}} \cdots \Omega_{i, j_{1}}^{2 j_{2}} \Gamma_{i, 0}\right)^{1 / 2^{t}} \bmod N_{i}\right]_{-1}
$$

where $\Omega_{i, j_{1} \ldots j_{l}}=<f_{\Omega}\left(C\left\|j_{1}\right\| \cdots\left\|j_{l}\right\| N_{i}\right)>_{1}$.

$P$ sends $\left(I_{i}, N_{i}, X_{i, j_{1} \ldots j_{i}}\right)(i=1, \ldots, K / 2)$ and $(B, C)$ to $V$.

Note: The above calculation of $X_{i, j_{1} \cdots j_{1}}$ is based on the following algoritlum:

$$
X_{i, j_{1} \cdots j_{t}}=\left[\Gamma_{i, j_{1} \cdots j_{t}}^{1 / 2} \bmod N_{i}\right]_{-1}
$$

where

$$
\Gamma_{i, j_{1} \cdots j_{t+1}}=\left[\Omega_{i, j_{1} \cdots j_{l}}^{j_{1+1}} \Gamma_{i, j_{1} \cdots j_{l}}^{1 / 2} \bmod N_{i}\right]_{Q R}
$$

Step 3: $V$ verifies the validity of the signatures $B$ for $\left\{\left(I_{i}, N_{i}\right)\right\}$, and $C$ for $B$. $V$ computes $\Omega_{i, \ldots, \ldots}$ (if $i_{t+1}=1$ ) then verifies the validity of $r_{;}, \ldots t=1, \ldots K / ?$ ) anch that

$$
\begin{aligned}
& \left(X_{i, j_{1} \cdots j_{r}} / N_{i}\right)=-1 \text {, } \\
& X_{i, j_{1} \cdots j_{t}}^{2^{t}}=d_{i} \Omega_{i, j_{1} \cdots j_{t-1}}^{2^{t-1} j_{t}} \Omega_{i, j_{1} \cdots j_{t-2}}^{2^{t-2} j_{t-1}} \cdots \Omega_{i, j_{1}}^{2 j_{2}} f_{\Gamma}\left(C\|0\| N_{i}\right) \bmod N_{i},
\end{aligned}
$$

where $d_{i} \in\{ \pm 1, \pm 2\}(i=1, \ldots, K / 2)$. If thcy are valid, $V$ selects random bits, $E_{i, j_{1} \cdots j_{t}} \in\{0,1\}(i=1, \ldots, K / 2)$, and sends them to $P$. Otherwise $V$ halls this protocol. 
Step 4: $P$ computes

$$
Y_{i, j_{1} \cdots j_{l}}=\left[\Lambda_{i, j_{1} \cdots j_{l}}^{1 / 2} \bmod N_{i}\right]_{(-1)^{E_{i, j_{1} \cdots, j_{l}}}}
$$

Here,

$$
\Lambda_{i, j_{1} \cdots j_{l}}=<f_{\Lambda}\left(C\left\|j_{1}\right\| \cdots\left\|j_{l}\right\| \Lambda_{i}\right)>_{Q R} .
$$

Step 5: $V$ verifies that

$$
\begin{gathered}
\left(Y_{i, j_{1} \cdots j_{t}} / N_{i}\right)=(-1)^{E_{i, j_{1} \cdots j_{t}},} \\
Y_{i, j_{1} \cdots j_{t}}^{2}=d_{i}^{\prime} f_{\Lambda}\left(C\left\|j_{1}\right\| \cdots\left\|j_{i}\right\| N_{i}\right) \bmod N_{i},
\end{gathered}
$$

where $d_{i}^{\prime} \in\{ \pm 1, \pm 2\}(i=1, \ldots, K / 2)$. If rcrification succeds, $V$ accepts $P^{\prime}$ s messages as payment of the amount duc.

Note: To prevent bank $A$ from crediting an invalid shop's account in Part III, we can enhance the protocol as follows: IIcre, we simply write $E_{i}$ as $E_{i, j_{1} \ldots j_{i}}$. $V$ sclects a random value $E_{i}^{\prime}$, and sends $V^{\prime}$ 's identity $I D_{Y^{\prime}}$, time $T$, and $E_{i}^{\prime}(i=1, \ldots, K / 2)$ to $P$ in place of sending $E_{i} . V$ computes $\left(E_{1}, \ldots, E_{K / 2}\right)=h\left(I D_{V}\|T\| E_{1}^{\prime} \ldots E_{K / 2}^{\prime}\right)$, where $h$ is a onc-way function whose output is uniformly random. $P$ also computes $E_{i}(i=1, \ldots, K / 2)$.

Part IV.

For bank $A$ to credit $V$ 's account by the appropriate amount, $V$ sends the history of Part IIl of this protocol, $H$, to $A$, which credits $V$ 's account. After checking the validity of $H$, bank $A$ must store $H$ in its databasc. If $A$ finds an invalid payment, $A$ reveal the secret information $S_{i}$ of costomer $P$ who is responsible for the invalid payment from $I I$ and the related history.

(End of Protocol 1)

Note 1: Since bank $A$ has already known $K / 2$ pieces of $S_{i}$ in Part I (e.g., $S_{K / 2+1}$, $\left.\ldots, S_{K}\right),(K / 2+1)$ pieces of $S_{i}$ shown by $A$ are the evidence of the invalid payment by a customer.

Note 2: Bank $A$ can store $H$ will dividing it into two parts, $H_{1}$ and $H_{2} . H_{1}$ is uscd to check the invalid payment, and $H_{2}$ is to compute $S_{i}$ when $A$ finds an invalid payment. $H_{1}$ consists of the hashed value of $C$ and the nodes corresponding to the payment. IIerc, the hashed value of $C$ is the searching key in the database, and $I_{1}$ can be very short (c.g, 10 bytes). On the other hand, $I_{2}$ is almost same as $H$, and is pointed from $H_{1}$. Therefore, $H_{1}$ can be stored in a database which is easy of access, while $J_{2}$ can be stored in a device such as a magnetic tape and a laser lisk, which is not easy of access but has big capacity. $H_{1}$ and $H_{2}$ (especially $H_{2}$ ) can be stored in a distributed manner.

\subsection{Correctness}

Here, we show bricfly that Protocol 1 satisfies the five critcria of (a) Independence, (b)Sccurity, (c) Privacy, (d)Off-line payment, and (f)Dinishilily. Among them, critcria (a) and (d) are clearly satisfied. Therefore, we show that the other three crilcria are satisfied.

- Privacy: First, if the customer accuratcly follows the protocol, even the coalition of bank $A$ and store $V$ cannot get any knowledge about the identity of $P$ with non-negligible probability, assuming that factoring is difficult for $A$ and $V$. 
- Dividalility: As shown in Subsection 2.2, if thrce restrictions on the usage of the hicrarchical structure table are salisfied, then the dividability condition is satisfied. (In the next item (security), we will show that the second and third restrictions are securely realized. The first restriction can be clcarly realized as a protocol.) Then, when $R$ is the ratio of the value of an clectronic bill, $C,(\mathrm{c} . \mathrm{g}, \$ 1000)$ to the minimum unit of payment (c.g., 1 cent), then the processing and communication amounts for payment are in proportion to $\log _{2} R$.

- Sccurity: First, we show that the third restriction of the hicrarchical structure table (Subscction 2.2) is sccurely realizcd. If customer $P$ uses any part of $C$ (any node of the hicrarchical structure table of $C$ ) more than once, bank $A$ can obtain the identity of $P$ with overwhelming probability, since the Williams integer $N$ can be factored in polynomial-time from $\left[x^{1 / 2} \bmod N\right]_{1}$ and $\left[x^{1 / 2} \bmod N\right]_{-1}$, and since $V$ challenges $P$ randomly using $\Lambda$ table, along with the cut-and-choose methodology. Next, we show that the second restriction of the hicrarchical structure table (Subsection 2.2 ) is securely rcalizcd. Here, for easily understanding, we use the simple example, where the value of $C$ is $\$ 100$, and $P$ pays $\$ 75$ to $V$ (Figure 1, 2, and 3 ). Notc that the cut-and-choose methodology is also implicitly crucial in assuring correctness, althongh we omit a detailed explanation here (roughly, thanks to this methodology, we can assume that $I_{i}, N_{i}$ are correctly gencrated).

First, we show that the first restriction is satisfied: that is, when nodes $\Gamma_{00}, \Gamma_{010}$ are used, then all descendant and ancestor nodes of thesc nodes, $\Gamma_{0}, \Gamma_{000}, \Gamma_{001}$, and $\Gamma_{01}$, cannot be used. When $\Gamma_{00}$ is used, $P$ sends $X_{i, 00}=\left[\Gamma_{i, 00}^{1 / 2} \bmod N_{i}\right]_{-1}(i=1, \ldots, K / 2)$ to $V$ (finally to $A$ ). Then, if $P$ uses $\Gamma_{000}, P$ sends $X_{i, 000}=\left[\Gamma_{i, 000}^{1 / 2} \bmod N_{i}\right]_{-1}$ $(i=1, \ldots, K / 2)$. Since $\left[\Gamma_{i, 00}^{1 / 2} \bmod N_{i}\right]_{1}=X_{i, 000}^{2} \bmod N_{i}, A$ can factor $N_{i}$ from $X_{i, 00}$ and $X_{i, 000}^{2} \bmod N_{i}$ (then, the identity of $P$ is revealcd). Similarly, if $\Gamma_{0}$ or $\Gamma_{001}$ is used with $\Gamma_{00}$, or if $\Gamma_{0}$ or $\Gamma_{01}$ is used with $\Gamma_{010}$, then the identity of $P$ is revcaled. Thercfore, when $\Gamma_{00}, \Gamma_{010}$ are used, then $\Gamma_{0}, \Gamma_{000}, \Gamma_{001}$, and $\Gamma_{01}$, cannot be used, with conccaling the identity of $P$.

Finally, we show the neccssity of $\Omega$, using a simple example. Ass ume that $\Omega_{i, j_{1} \ldots j_{1}}$ is a constant value, e.g., 3. Then, in Figure $3, \Gamma_{01}=3\left(\Gamma_{0}\right)^{1 / 2}$, where we omitt the suffix of $i$ and $\bmod N_{i}$, for simplicity. So, when a customer uses the nodes of $\Gamma_{00}$ and $\Gamma_{01}$, he opens the values of $X_{00}=\left(\Gamma_{0}\right)^{1 / 4}$ and $X_{01}=\left(3\left(\Gamma_{0}\right)^{1 / 2}\right)^{1 / 2}=3^{1 / 2}\left(\Gamma_{0}\right)^{1 / 4}$, where the jacobi symbol values of $X_{00}$ and $X_{01}$ are -1 . Then, the shop can obtain $3^{1 / 2}$ by calculating $X_{01} / X_{00}$, where the jacobi symbol of this value is 1 . The same situation occurs when the customer uses the nodes of $\Gamma_{000}$ and $\Gamma_{001}$, and so on. Therefore, suppose that a customer uses $\Gamma_{000}, \Gamma_{001}, \Gamma_{010}$, and $\Gamma_{0110}$, whose usage is valid. (So, he opens $X_{000}, X_{001}, X_{010}$, and $X_{0110}$.) Then, the shop can calculate $A=3^{1 / 2}$ by $X_{001} / X_{000}$, and also calculatc the valuc of $X_{011}$ by $A X_{010}$. Therefore, the shop can factor $N$ by using the values of $X_{011}$ and $\left(X_{0110}\right)^{2}$, where the jacobi symbol of $X_{011}$ is -1 and that of $\left(X_{0110}\right)^{2}$ is 1 . Thus, the shop can know the customer's ID, although the customer uses the nodes validly.

\section{Transferable Universal Electronic Cash}

In this section, we propose an electronic cash scheme satisfying the criterion of (e) Tmnsferability in addition to the other five criteria. 


\section{Protocol 2. (Transferable universal electronic cash)}

This protocol is constructed based on Protocol 1. To simplify the description of this protocol, we suppose an example similar to that in Section 3 , where $C$ is worth $\$ 100$, customer $P_{1}$ who has spent $\$ 75$ transfers the remaining $\$ 25$ to customer $P_{2}$, and $P_{2}$ uses $\$ 25$ at shop $V$.

Part I.

When customers $P_{1}$ and $P_{2}$ open their accounts at bank $A, A$ issues electronic licenses $B^{(j)}$ to a customer $P_{j}(j=1,2)$. Hereafter, in this protocol, $x^{(j)}$ means $x$ of $P_{j}$, where variable $x$ follows the definition in Protocol 1.

Part II.

Suppose that customer $P_{1}$ has bank $A$ issue an elcctronic bill worth $\$ 100, C$.

Part III.

To transfer $C$ to another customer $P_{2}, P_{1}$ and $P_{2}$ procceds as follows:

(Step 1) $P_{2}$ takes the role of $V$ in Protocol 1 as $P_{1}$ pays shop $P_{2} \$ 25$ (corresponding to node $\Gamma_{011}$ ) (Part III of Protocol 1).

(Step 2) $P_{1}$ sends certification $T$ that denotes the transfer of $C$ from $P_{1}$ to $P_{2}$. For example, $P_{1}$ sends a (Raljin scheme) digital signature $T=\left(\left\langle g\left(C\|011\| B^{(2)}\right\rangle_{Q R}\right)^{1 / 2} \bmod N_{1}^{(1)}\right.$.

Part IV.

To pay shop $V \$ 25, P_{2}$ and $V$ proceed as follows:

(Step 1) $P_{2}$ scnds the history of Part III of this protocol, $H^{(1)}$, to $V . V$ checks the validity of $H^{(1)}$.

(Step 2) $P_{2}$ follows Part III of Protocol 1 with shop $V$ to pay $C$. Here, $P_{2}$ sends $V$ messages corresponding to nodes $\Gamma_{011}^{(2)}$ and $\Lambda_{011}^{(2)}$.

Part V.

To have bank $A$ credit $V$ 's account by $\$ 25, V$ sends the history of Part IV of this protocol, $H^{(2)}$, to $A$, which credits $V$ 's account. Bank $A$ must store $H^{(2)}$ in its database.

(End of Protocol 2)

\section{Performance Estimation}

We will briefly explain an example of the ncw cash system implementation. IIcre we assume that $K=40,\left|N_{i}\right|$ is 64 bytes, and the hicrarchical structure table has $1 i$ levels. We also assume that a bank issues a piece of cash worth $\$ 1000$ to customer Alice. Alice can disburse her cash in any way she pleases until the total expended equals $\$ 1000$. Then, she uses just 64 bytes of data for the electronic bill $(C)$ worth $\$ 1000$ and her proper data (electronic license, $B$ ) is about several kilobytes. Thus the total amount of data is small enough to be stored on typical smart cards. When she buys several articles (e.g., the total payment for them is \$334.36) at a store, her card transmits only 20 kilobytes on average. The computation time for generating the data representing the payment (e.g., \$331.36) that will be sent to the store is about several seconds, assuming the existence of a Rabin scheme chip of $30 \mathrm{Kbps}$ (kilo-bit per second). If the value of the payment is known in advance, the computation for the payment can be executed in advance. 


\section{Conclusion}

In this paper, we have proposed the first ideal untraccable clcctronic cash systcm, The customer can subdivide his cash balance, $C$ (dollars), into many picces in any way he plcascs until the total value of all subdivided picce cquals $C$. A smart card cquipped with a Rabin sclocme clip and the distributed database system for a bank to store $H_{1}$ and $H_{2}$ should be implemented efficiently to realize the universal clectronic cash system. From a theoretical view point, it remains open to construct an unconditionally untraccable universal electronic cash system.

\section{Acknowledgments}

Authors wish to thank A. Ilerzberg, S. Kurihara, K. Sakurai and A. Shamir for their useful discussions.

\section{References}

[Ber] E.R.Berlckamp, "Factoring Polynomials over Jarge Finitc Fields," Math. Comp., Vol.24, No.111, pp.713-735 (1970)

[B1] M.Blum, "Coin flipping by tclephonc", IEEE, COMPCON, pp.133-137 (1982)

[Ch] D.Chaum, "Sccurity without Identification: Transaction Systems to Make Big Brother Obsoletc," Comm. of the ACM, 28, 10, pp.1030-1014 (1985)

[CFN] D.Chaum, A.Fiat and M. Naor, "Untraceable Electronic Cash," Proc. of Crypto'88, pp.319-327 (1988)

[CW] J.L.Carter and M.N.VVegman, "Universal Classes of IIash Functions," Journal of Computer and System Scicnces, 18, pp.143-154 (1979)

[Da] I.B.Damgård, "Payment Systems and Credential Mechanisms with Provable Security Against Abusc by Individuals," Proc. of Crypto'88, pp.328-335 (1988)

[EGL] S.Even, O.Goldrcich, A.Lempel: "A Randomized Protocol for Signing Contracts", Proc. of Crypto'82, pp.205-210 (1982)

[EGY] S.Even, O.Goldreich, Y'.Yacobi: “Electronic IVallet", Proc. of Crypto'83, pp.383386 (1983)

[FOM] A.Fujjoka, T.Okamoto, S.Miyaguchi: "ESICN: An Efficient Digital Signature Implementation for Smart Cards", to appear in Proc. of Eurocrypt'01

[H] B.Hayes, "Anonymous Onc-Time Signatures and Flexible Untraccable Elertronic Cash," Proc. of Auscrypt 90, pp.294-305 (1990)

[OkOh1] T.Okamoto, and K.Ohta "Disposable Zcro-Knowledge Authentications and Their Applications to Untraccable Electronic Cash," Proc. of Crypto'89, pp.181496 (1989) 
[OkOh2] T.Okamoto, and K.Ohta "Divertible Zcro-Knowiedge Interactive Proofs and Commutalive Random Self-Reducible," Proc. of Eurocrypt'89 (1989)

[PW] B.Pfitzmann, M.Waidner, "Ilow to Break and Repair a "Provably Sccure" Untraccable Payment System," to appear in Proc. of Crypto'91

[R] M.O.Rabin, "Digitalized Signatures and Public-Key Functions as Intractable as Factorization," Tcch. Rep., MTT/LCS/TR-212, MIT Lab. Comp. Sci., (1979)

[W] H.C.Williams, "A Modification of the RSA Public-Ficy Encryption Procedurc," IEEE Trans. on Information Theory, Vol.IT-26, No.6, pp.726-729 (1980) 